\title{
Geração de Sequências Curriculares Adaptativas baseada em Computação Evolucionária: Estado da Arte e Tendências
}

\author{
Marcelo O. C. Machado ${ }^{1}$, Jairo F. Souza ${ }^{1}$, Eduardo Barrére ${ }^{1}$ \\ ${ }^{1}$ Programa de Pós Graduação em Ciência da Computação - \\ Universidade Federal de Juiz de Fora (UFJF) \\ 36.360-900 - Juiz de Fora - MG - Brasil \\ \{marcelomachado, jairo.souza, eduardo.barrere\}@ice.ufjf.br
}

\begin{abstract}
For effective adoption of e-Learning systems, the student's needs and context must be taken into consideration when delivering content. One of the most interesting challenges in this area is the selection of an Adaptive Curriculum Sequencing that provides individualized instruction. Such a challenge is known in the literature as an NP-hard problem, causing frequent use of metaheuristics, particularly of Evolutionary Computing, to approximate the best solution. In this paper a review of the lastest works in this field of research is presented with a comparison model according to the variables used for the sequencing.
\end{abstract}

Resumo. Para efetiva adoção dos sistemas de e-Learning, as necessidades e o contexto do aluno precisam ser levados em consideração na entrega de conteúdo. Assim, um dos desafios mais interessantes da área é a seleção de uma Sequência Curricular Adaptativa que forneça um ensino individualizado. Tal desafio é conhecido na literatura como um problema NP-Difícil, causando uma frequente utilização de meta-heurísticas, particularmente de Computação Evolucionária, para aproximar à melhor solução. Neste artigo é feita uma revisão dos últimos trabalhos neste campo de pesquisa, apresentando um modelo de comparação de acordo com as variáveis utilizadas para o sequenciamento.

\section{Introdução}

A efetiva utilização de sistemas de e-Learning depende da criação de soluções para permanência dos alunos nos cursos ofertados [Davis et al. 2016] e de uma correta administração dos materiais didáticos para serem disponibilizados de acordo com a necessidade de aprendizagem de cada aluno [Van Merriënboer and Ayres 2005]. Sistemas de e-Learning adaptativos buscam resolver esses problemas levando em consideração as informações de cada aluno, tais como suas necessidades e contextos, podendo assim, recomendar conteúdo didático de maneira individualizada [Pushpa 2012]. [Al-Azawei and Badii 2014] pontuam alguns dos objetivos de um e-Learning adaptativo: melhorar a assimilação de conteúdo, reduzir o esquecimento, motivar os alunos, orientar o melhor caminho de aprendizagem, tratar a sobrecarga cognitiva, reduzir o custo de aprendizagem e melhorar a usabilidade dos sistemas. Isso significa que a adaptatividade pode desempenhar o papel de promover e melhorar a aprendizagem.

[Premlatha and Geetha 2015] indicam que existem diferentes níveis de adaptação, dentre eles o baseado na sequência curricular. A ideia desse nível é auxiliar o sistema a 
VI Congresso Brasileiro de Informática na Educação (CBIE 2017)

Anais do XXVIII Simpósio Brasileiro de Informática na Educação (SBIE 2017)

encontrar a melhor sequência de conteúdos educacionais que satisfaça as necessidades de um aluno. Uma das principais dificuldades de um e-Learning que se adapta a esse nível é definir os diferentes parâmetros que vão representar os alunos e os conteúdos educacionais. Assim, vários trabalhos na última década exploraram soluções utilizando diversos atributos [Wang and $\mathrm{Wu}$ 2011]. Embora outras nomenclaturas sejam adotadas para representar a sequência curricular, neste trabalho será adotado o termo Sequência Curricular Adaptativa (SCA). Nos últimos anos diversos trabalhos utilizaram abordagens de Computação Evolucionária (CE) para selecionar uma SCA que forneça um ensino individualizado. Isso acontece, principalmente, por se tratar de um problema de otimização combinatória pertencente a classe de problemas NP-Difícil [Acampora et al. 2011] que, de maneira clássica, são tratados utilizando heurísticas e meta-heurísticas.

Dada a importância da seleção da SCA em um e-Learning adaptativo e a crescente utilização de CE para aproximar a melhor solução desse problema, este artigo faz uma revisão de trabalhos que abordaram esse tema nos últimos anos. Então, é apresentado um modelo de comparação de parâmetros de adaptação, abordagem de CE utilizada, tipo de sequenciamento e a representação do Caminho de Aprendizagem (CA). O estudo permite compreender melhor o estado da arte na área, apoiando novos pesquisadores a criarem novas propostas e implementações em sistemas de $e$-Learning adaptativos.

O restante do artigo está organizado da seguinte forma: a seção 2 contextualiza o problema da SCA; a seção 3 apresenta os trabalhos relacionados; a seção 4 apresenta os trabalhos mais atuais da área, bem como um modelo de comparação dos mesmos de acordo com a maneira que a SCA é tratada; a seção 5 apresenta as conclusões do trabalho.

\section{Sequência Curricular Adaptativa}

$\mathrm{O}$ e-Learning adaptativo pode quebrar o famoso paradigma de ensino presencial onde "one size fits all", ou seja, um conceito é ensinado da mesma maneira, com os mesmos conteúdos educacionais, a todos os alunos [Acampora et al. 2011]. Portanto, tratar o problema da SCA que considere o modelo do aluno se mostra como uma das pesquisas mais interessantes e crucial em sistemas de aprendizagem online [Muhammad et al. 2016].

[Brusilovsky et al. 1999] se referem a essa sequência como um Sequenciamento Curricular (ou Planejamento Instrucional) cujo objetivo é fornecer ao aluno a ordenação mais adequada de unidades e tarefas de conhecimento (exemplos, perguntas, problemas, etc). O objetivo é ajudar o aluno a encontrar um "caminho ideal" de aprendizagem, maximizando a compreensão, bem como a eficiência no aprendizado.

[Kardan et al. 2015] indica que a SCA deve levar em consideração as características dos alunos, as informações dos materiais didáticos e o CA. Portanto, a SCA pode ser vista como uma função $f(a, d, c) \rightarrow S$ que recebe como parâmetros o modelo do aluno $a$, as informações dos materiais didáticos $d$ e as informações do CA $c$. Essa função tem como retorno a sequência $s \in S$ que melhor se aproxima do modelo do aluno (os trabalhos desta revisão satisfazem essa definição). O CA é uma representação do conteúdo programático a partir de interligações de seus tópicos. Por exemplo, para um curso de Cálculo, um CA possível seria: Números Reais, Funções, Limite de uma Função e Continuidade, Derivada e Aplicações da Derivada, onde os tópicos estão relacionados por ordem de pré-requisito ou por especialização. O CA pode ser gerado por especialistas ou de forma automática. Recentemente, trabalhos, tais como 
VI Congresso Brasileiro de Informática na Educação (CBIE 2017)

Anais do XXVIII Simpósio Brasileiro de Informática na Educação (SBIE 2017)

[de Marcos et al. 2011, Sharma et al. 2012] focaram na construção automática do CA.

A SCA é considerada um problema da classe NP-Difícil [Acampora et al. 2011], sobretudo por envolver diversas restrições a serem satisfeitas a fim de atender as necessidades dos estudantes. Dado um programa contendo $N$ conceitos a serem abordados, temse então $N$ ! sequências que poderiam ser selecionadas (sem considerar as subsequências). O espaço conceitual presente nos repositórios tende a aumentar com o passar do tempo fazendo com que as permutações de $N$ sejam cada vez maiores. Portanto, a seleção da SCA se torna ainda mais difícil, sobretudo, quando os parâmetros do aluno e dos materiais didáticos devem ser levados em consideração.

A SCA pode ter, por exemplo, um sequenciamento Individual ou Social. Além disso, o modelo do aluno é diferente a cada novo trabalho na literatura. Portanto, no modelo de comparação apresentado nesta revisão serão consideradas dimensões que representam as diferentes maneiras que a SCA foi abordada na literatura.

\section{Trabalhos Relacionados}

Por se tratar de um dos problemas mais populares em e-Learning adaptativo, diversos trabalhos relacionados a SCA foram desenvolvidos, principalmente, nas duas últimas décadas. Nesse cenário, algumas revisões foram realizadas buscando analisar o estado da arte. As revisões encontradas foram publicadas a partir do ano 2010 e apresentam os principais aspectos relacionados aos sistemas de $e$-Learning adaptativos.

[Khamparia and Pandey 2015] faz uma revisão ampla com relação aos problemas relacionados aos sistemas de e-Learning adaptativos. Essa revisão divide os trabalhos de acordo com os métodos utilizados nas soluções dos problemas, são eles: Métodos Baseados no Conhecimento, Métodos de Inteligência Computacional e, por fim, ambos combinados. É possível perceber nesse trabalho que a SCA é o problema mais abordado na área. De maneira similar, [Muhammad et al. 2016] também apresenta um levantamento dos esforços de adaptação em e-Learning em trabalhos de 2008 até 2015, destacando a importância na adoção da SCA.

[Wong and Looi 2010] e [Pushpa 2012] demonstram que abordagens de Inteligência de Enxames foram utilizadas em e-Learning, sendo válido ressaltar a SCA. O primeiro apresenta trabalhos publicados até o ano de 2009, que utilizaram Otimização por Colônia de Formigas (OCF) e Otimização por Enxame de Partículas (OEP), enquanto o segundo apresenta trabalhos que utilizaram apenas $\mathrm{OCF}$, que foram publicados até o ano de 2011. [Wong and Looi 2010] cria um sumário dos trabalhos a partir de características das técnicas utilizadas nas abordagens de CE. Ao passo que, [Pushpa 2012] lista os trabalhos de acordo com o contexto do usuário. O objetivo principal dessas pesquisas é destacar as principais conquistas de pesquisa que foram realizadas nos últimos anos.

A revisão de [Al-Muhaideb and Menai 2011], concentrada-se exclusivamente no problema da SCA que foram tradados com abordagens de CE. Os autores apresentam trabalhos do ano 2002 até 2009 em um sumário dividido de acordo com o tipo de sequenciamento (Individual ou Social). A discussão do trabalho enfatiza as ferramentas necessárias para facilitar a reutilização de conteúdo de aprendizagem e o sequenciamento automatizado.

Apesar da abrangência das revisões de [Khamparia and Pandey 2015] e 
VI Congresso Brasileiro de Informática na Educação (CBIE 2017)

Anais do XXVIII Simpósio Brasileiro de Informática na Educação (SBIE 2017)

[Muhammad et al. 2016], a SCA não é o assunto principal, ainda que os autores afirmem que esse é um problema crucial em e-Learning adaptativos. Embora [Wong and Looi 2010] e [Pushpa 2012] apresentem trabalhos que utilizaram CE para a tratar a SCA, apenas trabalhos que utilizaram OEP e OCF foram destacados, o que não abrange todas as soluções recentes. Em [Al-Muhaideb and Menai 2011], os parâmetros utilizados para o sequenciamento não são explicitados, bem como são apresentados trabalhos sobre a geração do CA ao invés da SCA.

Este artigo se propõe a apresentar os trabalhos mais recentes, destacando abordagens de CE que foram apresentadas em outras revisões e as que ainda não foram analisadas, demonstrando assim o crescimento na utilização de CE para o problema da SCA. Além de tratar o tipo de sequenciamento, os trabalhos foram sumarizados considerando também os principais parâmetros do(s) aluno(s) e do material didático, a abordagem de CE utilizada e a representação do seu CA.

\section{Sequência Curricular Adaptativa utilizando Computação Evolucionária}

A seleção dos trabalhos revisados foi realizada a partir dos termos de busca "Curriculum Sequencing", "Learning Path Generation", "Personalized Course Generation"e "Instructional Planning". Neste ponto, é válido ressaltar que o termo "Learning Path" foi utilizado, pois, em diversos trabalhos, esse é referenciado como sinônimo da SCA. Entretanto, na maioria dos casos, esse abrange apenas a representação do domínio dos conceitos que são alocados em uma estrutura que permita caminhamento pelo conteúdo. Portanto, foi necessário filtrar esses trabalhos. Por exemplo, o trabalho de [de Marcos et al. 2011, Shmelev et al. 2015] que mantêm essa definição. O objetivo do filtro foi manter o escopo desta revisão, embora o conceito seja necessário como um dos parâmetros apresentados em nossa revisão (última coluna da Tabela 1). Apenas trabalhos relacionados com CE foram selecionados. Os termos foram pesquisados a partir das bases Google Scholar, IEEE e Scopus. De forma a expandir a rede de trabalhos que pudessem não ser encontrados a partir dos termos principais, foram utilizadas as técnicas de Backward Snowballing e Forward Snowballing. A técnica Forward Snowballing busca trabalhos que utilizem a lista inicial como referência, enquanto a técnica Backward Snowballing busca novos trabalhos nas referências da lista inicial. Foram selecionados apenas trabalhos a partir de 2010, pois as outras revisões citadas abordam trabalhos dos anos anteriores. Assim, de um conjunto inicial de cerca de 160 trabalhos, foram selecionados 17 trabalhos que são revisados nesta seção.

\subsection{Modelo de comparação}

Neste trabalho, foi definido um modelo de comparação contendo 6 dimensões, de acordo com características comuns às soluções existentes na literatura: informações intrínsecas do aluno, informações intrínsecas do material, informações extrínsecas do aluno, tipo de sequenciamento e representação do CA.

Como parâmetros intrínsecos dos alunos, foram definidos a Intenção do aluno (I), que representa a finalidade de um aluno ao acessar o e-Learning. Por exemplo, obtenção de uma introdução geral do assunto ou relacionada ao trabalho de pesquisa. Tem-se também, o Tipo do aluno (A), o qual representa um grupo de características que definem as preferências de aprendizagem de cada aluno. A exemplo desse grupo de ca- 
VI Congresso Brasileiro de Informática na Educação (CBIE 2017)

Anais do XXVIII Simpósio Brasileiro de Informática na Educação (SBIE 2017)

racterísticas tem-se o Estilo de Aprendizagem (EA) [Agarwal et al. 2016] e o domínio cognitivo da Taxonomia de Bloom [Dharshini et al. 2015].

Os parâmetros intrínsecos do material didático possuem 5 características. A dificuldade do conceito (D) representa o grau de complexidade de um conceito, podendo ser ajustado por especialistas ou gerado a partir de testes. O tempo médio de aprendizagem (TM) normalmente representa um valor médio que os alunos deveriam gastar para aprendizado de um conceito. Esse tempo pode ser adicionado por especialistas ou capturados de metadados. O peso de um conceito (P) representa o grau de importância daquele conceito para o curso. Esse valor é normalmente ajustado por especialistas. A relação entre conceitos (C) que pode ser capturada do CA ou a partir da correlação entre os resultados dos alunos em pré-testes. A relação entre unidades e conceito (U) representa o grau de relevância entre a unidade selecionada e o conceito a ser ensinado. O tipo do material (M) representa a mídia de representação do material didático seja esse texto, áudio, vídeo etc. Esse pode representar ainda um teste, explicação, etc.

Os parâmetros extrínsecos do aluno dizem respeito às informações do ambiente ou do CA que estão relacionadas com o usuário. O nível de conhecimento de um conceito (N) representa o nível de maestria de um aluno perante a um conceito. Geralmente esse nível é calculado a partir de testes ou ainda capturado a partir de logs de comportamento do aluno no sistema. O tempo de aprendizagem (T) representa uma relação do nível de maestria do aluno e o tempo de aprendizado associado ao conceito. A qualidade de conexão (Q) representa a quantidade de banda de rede disponível no momento que o usuário está acessando o e-Learning. A relação entre material didático e aluno (R) representa o grau de pertinência de um material didático para um aluno. Esse valor a princípio é gerado aleatoriamente e depois é ajustado no decorrer do curso.

Devido a limitação de espaço, as abordagens de CE não serão detalhadas neste trabalho. Todavia, maiores informações sobre as meta-heurísticas (OCF, OEP e Algoritmo Genético - AG) utilizadas no problema da SCA podem ser encontradas em [Al-Muhaideb and Menai 2011]. Informações sobre o Algoritmo de Busca Harmônica $(\mathrm{ABH})$ podem ser encontradas em [Hnida et al. 2016]. Detalhes do algoritmo evolucionário PBIL (Population Based Incremental Learning) estão em [Wan and Lyu 2014] e [Acampora et al. 2011] esclarece sobre o Algoritmo Memético (AM).

Há dois grupos para o sequenciamento: (SI) Sequenciamento Individual, o qual considera apenas as informações do aluno que receberá a SCA e (SS) Sequenciamento Social, onde as experiências de alunos passados podem beneficiar alunos futuros.

$\mathrm{Na}$ literatura relacionada ao $\mathrm{CA}$, há diversas representações. Porém, apenas as utilizadas no escopo dos trabalhos desta revisão serão discutidas. O CA normalmente é alocado a uma estrutura que permita o caminhamento na trilha dos conceitos de um curso. Uma das representações mais comuns para este parâmetro é em uma estrutura de grafos. Nesse caso, cada nó denota um grupo de conceitos, onde cada um desses grupos consiste de várias unidades de um mesmo conceito, podendo representar diversas profundidades de dificuldade. O grafo pode ser criado a partir de ontologias de domínio e dos metadados dos materiais didáticos. Outra representação do CA é a estrutura de árvore, a qual o princípio determinante é que há uma hierarquia entre os conceitos. Matrizes também são utilizadas, de forma que os conceitos são correlacionados com base nas respostas dos 
VI Congresso Brasileiro de Informática na Educação (CBIE 2017)

Anais do XXVIII Simpósio Brasileiro de Informática na Educação (SBIE 2017)

alunos a um pré-teste. Alguns trabalhos criaram o CA de forma automática utilizando o SCORM (Sharable Content Object Reference Model), a qual designa o conjunto de padrões e especificações que compõem o protocolo de comunicação entre os objetos de aprendizagem. O SCORM utiliza o modelo LOM (Learning Object Metadata) que descreve os materiais didáticos. O LOM pode ser utilizado em conjunto com RDC (Reusable Definition of Competency) definindo uma sequência entre os materiais didáticos baseada no conceito de competências ([Dharshini et al. 2015]).

\subsection{Resultados e análise}

A Tabela 1 apresenta o sumário dos trabalhos analisados em relação à SCA. Nesta tabela, PIA representa parâmetros intrínsecos do aluno, PMD indica parâmetros do material didático, PEA denota os parâmetros extrínsecos do aluno, TS significa o tipo de sequenciamento e, por fim, o tipo de representação do CA.

Tabela 1. Sumário dos trabalhos em relação à SCA

\begin{tabular}{|c|c|c|c|c|c|}
\hline Artigo & PIA & PMD & PEA & $\mathrm{CE}$ & TS CA \\
\hline [Bhaskar et al. 2010] & $\mathrm{I}, \mathrm{A}$ & $\mathrm{D}, \mathrm{C}, \mathrm{U}$ & Q & $\mathrm{AG}$ & SI Grafo \\
\hline [Acampora et al. 2011] & $\mathrm{I}, \mathrm{A}$ & $\mathrm{D}, \mathrm{TM}, \mathrm{M}$ & $\mathrm{N}, \mathrm{T}$ & AM & SI Grafo \\
\hline [Jebari et al. 2011] & - & $\mathrm{D}, \mathrm{C}, \mathrm{P}, \mathrm{U}$ & $\mathrm{N}$ & $\mathrm{AG}$ & SI Grafo \\
\hline [Wang 2011] & A & $\mathrm{D}$ & $\mathrm{N}$ & OCF+Fuzzy & SS Grafo \\
\hline [Vazquez et al. 2011] & - & $\mathrm{D}, \mathrm{P}$ & $\mathrm{N}$ & $\mathrm{OCF}+\mathrm{RB}$ & SS Grafo \\
\hline [Chu et al. 2011] & - & $\mathrm{D}, \mathrm{TM}, \mathrm{P}, \mathrm{U}$ & $\mathrm{N}, \mathrm{T}, \mathrm{R}$ & OEP & SS SCORM \\
\hline [Riad et al. 2012] & A & $\mathrm{D}, \mathrm{M}$ & $\mathrm{N}$ & OCF & SS Grafo \\
\hline [Li et al. 2012] & - & $\mathrm{D}, \mathrm{TM}, \mathrm{P}, \mathrm{U}$ & $\mathrm{N}, \mathrm{T}, \mathrm{R}$ & AG e OEP & SS Árvore \\
\hline [Tan et al. 2012] & - & $\mathrm{D}, \mathrm{TM}$ & $\mathrm{N}, \mathrm{T}$ & $\mathrm{AG}$ & SI Grafo \\
\hline [Sharma et al. 2012] & - & $\mathrm{D}$ & $\mathrm{N}, \mathrm{T}$ & OCF & SS Grafo \\
\hline [Chakraverty et al. 2012] & $\mathrm{I}$ & $\mathrm{D}, \mathrm{TM}$ & $\mathrm{N}, \mathrm{T}, \mathrm{R}$ & $\mathrm{OCF}$ & SS Grafo \\
\hline [Kardan et al. 2014] & - & $\mathrm{D}$ & $\mathrm{N}$ & $\mathrm{OCF}$ & SS Grafo \\
\hline [Wan and Lyu 2014] & A & $\mathrm{P}, \mathrm{C}, \mathrm{U}, \mathrm{M}$ & - & PBIL & SI Ajustado \\
\hline [Dharshini et al. 2015] & A & $\mathrm{D}$ & - & $\mathrm{OCF}$ & SI SCORM+RDC \\
\hline [Pires and Cota 2015] & $\mathrm{I}$ & $\mathrm{D}$ & $\mathrm{N}$ & $\begin{array}{l}\text { AG+Função } \\
\text { estatística }\end{array}$ & SI SCORM \\
\hline [Hnida et al. 2016] & - & $\mathrm{D}, \mathrm{C}, \mathrm{TM}, \mathrm{P}$ & $\mathrm{N}$ & $\mathrm{ABH}$ & SI Matriz \\
\hline [Agarwal et al. 2016] & $\mathrm{I}, \mathrm{A}$ & $\mathrm{M}$ & $\mathrm{N}$ & OCF+Fuzzy & SS Grafo \\
\hline
\end{tabular}

A lista inicial de trabalhos demonstra que o e-Learning adaptativo é um tema que continua sendo trabalhado, especialmente em termos ligados à SCA. Os trabalhos selecionados apontam que diversos parâmetros tanto do aluno quanto do material didático e do CA foram explorados. O nível de conhecimento do aluno foi o parâmetro mais utilizado na dimensão do aluno, demonstrando que a adaptação deve levar em consideração o conhecimento prévio do aluno, aumentando a eficiência na aprendizagem. Embora o trabalho de [Dharshini et al. 2015] não utilize tal parâmetro de forma explicita, a intenção de aprendizagem é demonstrada a partir de um dos níveis do domínio cognitivo da Taxonomia de Bloom. Por outro lado, pode-se perceber que o contexto de infraestrutura do aluno não é largamente explorado, visto que apenas um dos trabalhos considerou o 
VI Congresso Brasileiro de Informática na Educação (CBIE 2017)

Anais do XXVIII Simpósio Brasileiro de Informática na Educação (SBIE 2017)

parâmetro "Qualidade de Conexão", embora exista a impressão de que esse parâmetro seja fundamental para a Educação a Distância. É possível perceber, também, que quase metade dos trabalhos não utilizam nenhum dos parâmetros intrínsecos relacionados aos alunos. Indicando que, apesar da tentativa de adaptabilidade voltada ao aluno seja o assunto chave para a construção da SCA, as informações que os definem e os distinguem ainda são pouco utilizadas. Uma das explicações para tal, está relacionada à dificuldade de relacionamento desses parâmetros com o domínio conceitual, sendo difícil, por exemplo, associar um EA a um material específico contido em um repositório. Para trabalhos futuros nessa área, seria interessante que esses parâmetros fossem mais explorados, bem como indicam os trabalhos futuros da maioria dos trabalhos aqui revisados.

O parâmetro mais utilizado na dimensão do material didático está relacionado à dificuldade. Isso era esperado, dado que é necessário ter um relacionamento desse parâmetro com a dificuldade do aluno para se compreender o nível de maestria de aluno.

As meta-heurísticas OCF e AG continuam sendo as abordagens de CE mais utilizadas para o problema da SCA ([Al-Muhaideb and Menai 2011]). É possível perceber uma relação com o tipo de sequenciamento, onde, na maioria dos trabalhos, OCF está relacionada ao SS, assim como AG está relacionado ao SI. Devido comportamento está relacionado à fundamentação dessas meta-heurísticas. Com AG, normalmente busca-se encontrar a melhor população de materiais didáticos para um aluno e, com OCF, o comportamento dos alunos (formigas) interferem no caminho dos demais. Porém, em [Dharshini et al. 2015], as formigas representam os materiais didáticos e, em [Li et al. 2012], utiliza-se o feedback dos alunos para ajustar a dificuldade dos materiais, justificando assim a divergência. Essa relação mostra uma tendência no momento de utilização de uma abordagem em detrimento de outra.

[Li et al. 2012] utilizam duas abordagens de CE de forma paralela, pois um dos objetivos é prever qual tem o melhor desempenho de acordo com o crescimento do domínio conceitual. Essa é uma estratégia interessante pois a área carece de benchmarks para comparação das soluções. [Vazquez et al. 2011] utilizam Rede Bayesiana (RB) para prever a probabilidade de sucesso em compreender uma unidade através do histórico e dos perfis dos alunos. [Wang 2011] e [Agarwal et al. 2016] trabalharam com lógica Fuzzy para melhorar a seleção dos materiais didáticos de acordo com as informações da sequência encontrada pela abordagem de CE utilizada, levando em consideração o desempenho. Enquanto, [Pires and Cota 2015] empregam uma função estatística (Quiquadrado) que serve como uma pré-avaliação associada à função de fitness. Ou seja, técnicas já conhecidas estão sendo utilizadas em conjunto com abordagens de CE para melhorar a acurácia ou o desempenho da solução.

Duas abordagens de CE que não haviam sido citadas em trabalhos anteriores de revisão foram utilizadas: PBIL e ABH: [Wan and Lyu 2014] abordam a adaptabilidade utilizando PBIL visto que esse algoritmo é aplicado em problemas com altos requisitos computacionais. O trabalho faz uma comparação com um problema utilizando AG e indica que o PBIL tem melhores resultados de desempenho e acurácia. Por outro lado, [Agarwal et al. 2016] utilizam ABH pois essa assim como OEP e AG pertencem a uma classe de algoritmos não determinísticos cuja solução pode variar a cada execução. Assim, a qualidade da solução obtida depende da população inicial, dos parâmetros e operações escolhidos. AG e OEP requerem um conjunto de configurações. Além disso, AG precisa 
VI Congresso Brasileiro de Informática na Educação (CBIE 2017)

Anais do XXVIII Simpósio Brasileiro de Informática na Educação (SBIE 2017)

de codificação cromossômica, que é diferente de um problema para outro. Por outro lado, $\mathrm{ABH}$ poderia ser implementada diretamente, precisando apenas de parâmetros tais como a função de fitness e o critério de parada. Essa abordagem ainda retorna um conjunto de soluções, isto é, retorna SCAs ordenadas cuja melhor solução está mais acima. Esses dois últimos trabalhos, em conjunto com [Acampora et al. 2011], mostram através de suas soluções, que é válido explorar abordagens ainda não tão comuns na literatura de SCA.

A representação mais comum do CA utilizada foi a estrutura de grafos, valendo-se ressaltar que em alguns casos os grafos foram ajustados manualmente por especialistas. Para efetiva utilização das soluções apresentadas em sistemas tradicionais de $e$-Learning se faz necessário utilizar formas automáticas para criar/capturar o CA através dos padrões utilizados por esses sistemas. A exemplo, [Chu et al. 2011, Dharshini et al. 2015, Pires and Cota 2015] utilizam padrões de sistemas de e-Learning tradicionais, embora não citem a estrutura que foi utilizada para representação do CA.

\section{Conclusão}

Nesta revisão foram apresentados os trabalhos publicados a partir de 2010 que utilizaram abordagens de CE para aproximar a melhor SCA relacionada aos alunos, complementando as revisões publicadas anteriormente. Esses trabalhos foram sumarizados de acordo com um modelo de comparação composto por 6 dimensões contendo os parâmetros do aluno, material didático, caminho de aprendizagem, e abordagem de CE utilizada.

A partir do modelo, foi possível perceber os parâmetros que mais são levados em consideração no momento do sequenciamento. Neste ponto, é válido ressaltar que ainda existe uma demanda por trabalhos que abordem mais parâmetros intrínsecos dos alunos, bem como o contexto de infraestrutura. O modelo mostra também uma relação entre a abordagem de CE utilizada e o tipo de sequenciamento. É possível perceber, ainda, que técnicas já conhecidas, tais como lógica $F u z z y$, funções estatísticas e RB, foram utilizadas em conjunto com abordagens de $\mathrm{CE}$ na tentativa de melhorar a acurácia e desempenho das soluções. Portanto, outras técnicas e abordagens ainda poderão ser aplicadas para solução desse problema ao longo dos próximos anos, fazendo ainda mais urgente a criação de um benchmarking para comparação das soluções.

A partir desta revisão espera-se que os novos pesquisadores e desenvolvedores da área tenham uma visão geral dos últimos esforços nesse tema e, a partir daí, tenham um direcionamento no momento de criar suas soluções.

\section{Referências}

Acampora, G., Gaeta, M., and Loia, V. (2011). Hierarchical optimization of personalized experiences for e-learning systems through evolutionary models. Neural Computing and Applications, 20(5):641-657.

Agarwal, S., Goyal, M., Kumar, A., and Rajalakshmi, K. (2016). Intuitionistic fuzzy ant colony optimization for course sequencing in e-learning. In Contemporary Computing (IC3), 2016 Ninth International Conference on, pages 1-6. IEEE.

Al-Azawei, A. and Badii, A. (2014). State of the art of learning styles-based adaptive educational hypermedia systems (ls-baehss). International Journal of Computer Science \& Information Technology, 6(3):1. 
VI Congresso Brasileiro de Informática na Educação (CBIE 2017)

Anais do XXVIII Simpósio Brasileiro de Informática na Educação (SBIE 2017)

Al-Muhaideb, S. and Menai, M. E. B. (2011). Evolutionary computation approaches to the curriculum sequencing problem. Natural Computing, 10(2):891-920.

Bhaskar, M., Das, M. M., Chithralekha, T., and Sivasatya, S. (2010). Genetic algorithm based adaptive learning scheme generation for context aware e-learning. International Journal on Computer Science and Engineering, 2(4):1271-1279.

Brusilovsky, P. et al. (1999). Adaptive and intelligent technologies for web-based eduction. $K i, 13(4): 19-25$.

Chakraverty, S., Hans, S., and Mittal, V. (2012). Aco-driven personalized e-learning with perspectives and learning aims. In Proceedings of the International Conference on E-learning, E-business, Enterprise Information Systems, \& E-government, pages 359365.

Chu, C.-P., Chang, Y.-C., and Tsai, C.-C. (2011). Pc2pso: personalized e-course composition based on particle swarm optimization. Applied Intelligence, 34(1):141-154.

Davis, D., Chen, G., Hauff, C., and Houben, G.-J. (2016). Gauging mooc learners' adherence to the designed learning path. In EDM '16: 9th International Conference on Educational Data Mining, page ...

de Marcos, L., Martinez, J.-J., Gutiérrez, J.-A., Barchino, R., Hilera, J.-R., Oton, S., and Gutiérrez, J.-M. (2011). Genetic algorithms for courseware engineering. International Journal of Innovative Computing, Information and Control, 7(7):1-27.

Dharshini, A. P., Chandrakumarmangalam, S., and Arthi, G. (2015). Ant colony optimization for competency based learning objects sequencing in e-learning. Applied Mathematics and Computation, 263:332-341.

Hnida, M., Idrissi, M. K., and Bennani, S. (2016). Adaptive teaching learning sequence based on instructional design and evolutionary computation. In Information Technology Based Higher Education and Training (ITHET), 2016 15th International Conference on, pages $1-6$. IEEE.

Jebari, K., El Moujahid, A., Bouroumi, A., and Ettouhami, A. (2011). Genetic algorithms for online remedial education based on competency approach. In Multimedia Computing and Systems (ICMCS), 2011 International Conference on, pages 1-6. IEEE.

Kardan, A. A., Aziz, M., and Shahpasand, M. (2015). Adaptive systems: a content analysis on technical side for e-learning environments. Artificial Intelligence Review, 44(3):365-391.

Kardan, A. A., Ebrahim, M. A., and Imani, M. B. (2014). A new personalized learning path generation method: Aco-map. Indian Journal of Scientific Research, 5(1):17.

Khamparia, A. and Pandey, B. (2015). Knowledge and intelligent computing methods in e-learning. International Journal of Technology Enhanced Learning, 7(3):221-242.

Li, J.-W., Chang, Y.-C., Chu, C.-P., and Tsai, C.-C. (2012). A self-adjusting e-course generation process for personalized learning. Expert Systems with Applications, 39(3):3223-3232.

Muhammad, A., Zhou, Q., Beydoun, G., Xu, D., and Shen, J. (2016). Learning path adaptation in online learning systems. In Computer Supported Cooperative Work in Design (CSCWD), 2016 IEEE 20th International Conference on, pages 421-426. IEEE. 
VI Congresso Brasileiro de Informática na Educação (CBIE 2017)

Anais do XXVIII Simpósio Brasileiro de Informática na Educação (SBIE 2017)

Pires, J. M. and Cota, M. P. (2015). A new learning cognitive architecture using a statistical function and genetic algorithms: An intelligent new e-learning model. In $e$ Learning (econf), 2015 Fifth International Conference on, pages 1-8. IEEE.

Premlatha, K. and Geetha, T. (2015). Learning content design and learner adaptation for adaptive e-learning environment: a survey. Artificial Intelligence Review, 44(4):443465.

Pushpa, M. (2012). Aco in e-learning: Towards an adaptive learning path. International Journal on Computer Science and Engineering, 4(3):458.

Riad, B., Ali, S., Mourad, H., and Hamid, S. (2012). An adaptive learning based on ant colony and collaborative filtering. In Proceedings of the World Congress on Engineering, volume 2, pages 851-855.

Sharma, R., Banati, H., and Bedi, P. (2012). Adaptive content sequencing for e-learning courses using ant colony optimization. In Proceedings of the International Conference on Soft Computing for Problem Solving (SocProS 2011) December 20-22, 2011, pages 579-590. Springer.

Shmelev, V., Karpova, M., and Dukhanov, A. (2015). An approach of learning path sequencing based on revised bloom's taxonomy and domain ontologies with the use of genetic algorithms. Procedia Computer Science, 66:711-719.

Tan, X.-h., Shen, R.-m., and Wang, Y. (2012). Personalized course generation and evolution based on genetic algorithms. Journal of Zhejiang University SCIENCE C, 13(12):909-917.

Van Merriënboer, J. J. and Ayres, P. (2005). Research on cognitive load theory and its design implications for e-learning. Educational Technology Research and Development, 53(3):5-13.

Vazquez, J. M. M., Ramirez, J. A. O., Gonzalez-Abril, L., and Morente, F. V. (2011). Designing adaptive learning itineraries using features modelling and swarm intelligence. Neural Computing and Applications, 20(5):623-639.

Wan, S. and Lyu, C. (2014). Adaptive course generation based on evolutionary algorithm. In Information Science and Technology (ICIST), 2014 4th IEEE International Conference on, pages 168-171. IEEE.

Wang, F.-H. (2011). Personalized recommendation for web-based learning based on ant colony optimization with segmented-goal and meta-control strategies. In Fuzzy Systems (FUZZ), 2011 IEEE International Conference on, pages 2054-2059. IEEE.

Wang, S.-L. and Wu, C.-Y. (2011). Application of context-aware and personalized recommendation to implement an adaptive ubiquitous learning system. Expert Systems with applications, 38(9):10831-10838.

Wong, L.-H. and Looi, C.-K. (2010). A survey of optimized learning pathway planning and assessment paper generation with swarm intelligence. Intelligent Tutoring Systems in E-learning Environments: Design, Implementation and Evaluation, Hershey: IGI Global, pages 285-302. 